\section{Giving RSV some POP(G)}

\section{Tracey Baas, Associate Editor}

A group at National Jewish Health has shown that a low-cost pulmonary surfactant called palmitoyl-oleoyl-phosphatidylglycerol, already known to attenuate respiratory syncytial virus-induced inflammatory responses, also inhibits binding of the virus to lung epithelial surfaces. ${ }^{1}$ Although delivery and timing issues still need to be worked out, the surfactant's mechanism could offer a one-two punch to prevent or treat the infection.

Respiratory syncytial virus (RSV) is a leading cause of hospitalization in children younger than six months. Infection progresses from a mild upper airway condition that in most cases goes undiagnosed to a more serious and less accessible lower airway episode. Major risk factors leading to serious RSV infection, which in turn can cause acute respiratory distress syndrome (ARDS), are preterm birth, congenital heart defects and chronic lung disease.

The one approved drug for RSV is Synagis palivizumab from AstraZeneca plc's MedImmune LLC business unit. The anti-RSV antibody is indicated to prevent RSV in high-risk children. The average wholesale price is about $\$ 1,000$ per shot, which is given monthly through the RSV season. That price tag has limited its use to premature infants and precluded its application in full-term infants and young children. ${ }^{2}$

Indeed, the American Academy of Pediatrics' Committee on Infectious Disease revised its treatment guidelines last year, based on "issues of efficacy, cost, and evidence-based medicine," which could decrease the number of premature infants considered candidates for Synagis.

The most notable changes included the recommendations for infants of 32-35 weeks gestational age. Those infants that are three months old or less at the start of RSV season who also have at least one more risk factor are recommended to receive the prophylactic. The 2006 recommendations suggested that the infants be six months or less. ${ }^{3}$

The group at National Jewish Health has been looking for lowcost alternatives. Their strategy is to increase the levels of pulmonary surfactant, a lipoprotein mixture secreted by the lung that plays a role in host innate immunity. The surfactant they have their sights set on, palmitoyl-oleoyl-phosphatidylglycerol (POPG), can be obtained from suppliers for about $\$ 400$ per gram.

In September 2009, Dennis Voelker and colleagues at National Jewish Health reported that POPG decreased toll-like receptor 4 (TLR4) activation and inflammatory responses to lipopolysaccharide in the lungs of mice. ${ }^{4}$ Now, his team has shown that POPG directly binds RSV in vitro and reduces viral infection and lung pathology in vivo. ${ }^{1}$
In RSV-infected epithelial cell lines, POPG liposomes prevented both viral spread to neighboring cells and cell death. When the cells were treated with the liposomes prior to infection, RSV was unable to bind to the cell surface and RSV-induced proinflammatory cytokine production was reduced compared with what was seen using control phospholipids.

In mice, intranasal delivery of POPG liposomes plus RSV resulted in significantly lower viral infection and lung pathology than occurred with delivery of RSV alone $(p<0.01)$. Results were published in the Proceedings of the National Academy of Sciences.

"With RSV disease, virus replication and inflammation go hand in hand. If one compound could provide both antiviral and anti-inflammatory benefits, that would be extremely useful," said Leonard Krilov, chief of pediatric infectious diseases at Winthrop-University Hospital.

The recognition of a direct inhibitory role of POPG on RSV is important "because it identifies types of endogenous molecules that can now be used in a supplemental manner to suppress inflammation that has spiraled out of control" in conditions that can accompany RSV such as acute respiratory distress and also acute lung injury, said Voelker, senior author on the PNAS paper and a professor of biochemistry, molecular genetics and medicine at National Jewish Health.

He added that a detailed understanding of the action of POPG "will also provide a structural basis for designing POPG-like molecules with greater potency and selectivity."

\section{Modeling RSV}

Because the National Jewish Health team mixed virus and POPG outside the mouse and then delivered the mixture to the animals, the group lacks in vivo data on whether POPG has the potential to treat RSV rather than just prevent it. Thus, whereas Voelker's group wants to explore the use of POPG to both treat and prevent RSV, other researchers contacted by $S c i B X$ wanted to see the surfactant used to treat an RSV infection already underway in vivo.

"A more representative test of therapeutic pharmacological usefulness would be to treat challenged mice with POPG rather than mixing both virus and POPG prior to challenge," said Sonya Cyr, head of preclinical RSV research at GlaxoSmithKline plc's GlaxoSmithKline Biologicals North America unit.

Epithelial cells pretreated with POPG and then infected with RSV showed the reduced levels of proinflammatory cytokine production characteristic of an innate immune response. However, the PNAS article did not describe what happens to levels of proinflammatory cytokine production in RSV-infected cells treated with POPG, noted Asuncion Mejias, a principal investigator on RSV pathogenesis and host response at The Research Institute at Nationwide Children's Hospital.

Thus, Mejias told SciBX, "we don't really know, in vivo or in vitro, how this compound would be beneficial to the host immune response in an established RSV infection, which is what we see clinically every day. The effect of the compound in an established RSV infection needs to be explored in vivo." 
"The next step in the RSV field is going beyond prevention of infection [to] treatment of ongoing disease," said Bruce Keyt, CSO and VP of research at Trellis Bioscience Inc. He added that he would also like to see results from delayed administration of POPG following RSV inoculation.

Last month, Trellis granted MedImmune an exclusive worldwide license to develop and commercialize antibodies against RSV. The antibodies, which are in preclinical testing, were discovered using Trellis' CellSpot screening technology.

MedImmune did not return calls to comment on the findings.

\section{Taking POPG to the lung}

Voelker said his laboratory is already running postinfection treatment studies in mice and has plans for studies in larger animals. "We would like to perform some studies in primates and then proceed to Phase I trials," he said. "Since phosphatidylglycerol has already been given to humans in the form of therapeutic pulmonary surfactant preparations, we may be able to quickly move through clinical trials."

He said POPG could be given as a nasal spray for infections that are caught early enough so that the virus is still limited to the upper airway.

Jeffrey Kahn, chief of the Division of Pediatric Infectious Diseases at The University of Texas Southwestern Medical Center at Dallas, said it also will be important for the treatment to reach the lower lungs.

"RSV-infected children first present with subtle respiratory changes similar to those of a common cold. By the time inflammation and respiratory distress start to become apparent, resulting in a doctor's visit, the virus has been replicating for several days and has progressed to the lower airways," he said.

Voelker said lower lung delivery is a challenge but is not insurmountable. "It is also likely that POPG could be applied as a microparticulate powder that could be inhaled to treat infections in the alveolar compartment of the lung," he told SciBX.

"More uniform delivery of POPG throughout the lung might be better achieved when administered as a component of an exogenous therapeutic surfactant specifically designed to optimize intrapulmonary distribution and lung aeration," said Jan Mazela, senior clinical research fellow at Discovery Laboratories Inc., which is developing a surfactant product to prevent respiratory distress syndrome (RDS) in premature infants.

Leonard Weisman, professor of pediatrics at Baylor College of Medicine, said that in certain cases compounds can be delivered via intubation for mechanical ventilation. "About 2\% of RSV-infected children become hospitalized and about $10 \%$ of those are admitted to the ICU for mechanical ventilation," he noted.

The molecule also could have a home in the prophylaxis setting, assuming it can be maintained at an appropriate level in the airways.

"We do not yet know the turnover of POPG in vivo in mice," said Voelker. "We are currently engaged in such studies. Based upon respiratory rate, the turnover in mice is expected to be on the order of 1-3 hours. By comparison, the turnover of POPG in humans, based upon studies with exogenous surfactant instilled into premature newborns, is 30 hours." $^{5}$

Other groups have used combinations of pulmonary surfactants, including POPG, to treat RSV infection, acute lung injury (ALI) or ARDS with limited success. ${ }^{6-8}$

Voelker suspects this is a result of using surfactant mixtures rather than pure POPG. "Our studies lead us to the conclusion that the reason these preparations have not been effective is because they are produced in a manner that causes random mixing of POPG with other lipids," he said.

Although currently available commercial surfactants to treat preterm infants with RDS have low amounts of POPG, Charles Cochrane of The Scripps Research Institute noted that lucinactant, a synthetic peptide-based surfactant, "contains relatively high concentrations of POPG-about $25 \%$ of the formulation's total phospholipid content as an active pharmaceutical ingredient."

Cochrane, professor emeritus of immunology at Scripps, is the inventor of lucinactant, which Discovery Labs now is developing as Surfaxin. Surfaxin, a humanized lung surfactant, is under review for approval by the FDA to prevent RDS in premature infants. Last year, after a meeting with the agency, the company said it had established a regulatory path for Surfaxin. The same drug product is being developed in an aerosolized form called Aerosurf, which should allow earlier, noninvasive drug delivery.

Robert Segal, the company's CMO, noted that Surfaxin is in Phase II testing to treat acute hypoxic respiratory failure due to viral infection including RSV and influenza in children up to two years of age. This trial is being conducted in collaboration with the Pediatric Acute Lung Injury and Sepsis Investigators (PALISI) network, and data are expected this year.

Whether "the anti-inflammatory and antibacterial properties of lucinactant are directly related to the presence of POPG alone in the formulation remains unanswered," Segal added.

Similar to the observations by Voelker's team, lucinactant has been shown to suppress the production of proinflammatory cytokines IL-6 and IL- 8 in cultured human airway epithelium and a preterm lamb model of RDS. ${ }^{9}$

Ultimately, Segal said additional studies will be required to determine the optimal dose of POPG and to verify its anti-inflammatory role as both a prophylactic approach and treatment for RSV, ARDS and ALI.

"The work from Dr. Voelker's team is an important step in this direction," he said.

National Jewish Health has filed for a patent covering the use of POPG to treat RSV infection, ARDS and ALI. The application is available for licensing through National Jewish Health's IP office.

Baas, T. SciBX 3(2); doi:10.1038/scibx.2010.35

Published online Jan. 14, 2010

\section{REFERENCES}

1. Numata, M. et al. Proc. Natl. Acad. Sci. USA; published online Dec. 22, 2009; doi:10.1073/pnas.0909361107

Contact: Dennis Voelker, National Jewish Health, Denver, Colo. e-mail: voelkerd@njhealth.org

2. Usdin, S. BioCentury 15(9), A1-A9; Feb. 17, 2007

3. Krilov, L et al. Pediatrics 124, 1682-1684 (2009)

4. Kuronuma, K. et al. J. Biol. Chem. 284, 25488-25500 (2009)

5. Hallman, M. et al. Clin. Pharmacokinet. 26, 215-232 (1994)

6. Tibby, S. et al. Am. J. Respir. Crit. Care Med. 162, 1251-1256 (2000)

7. Luchetti, M. et al. Pediatr. Crit. Care Med. 3, 261-268 (2002)

8. Spragg, R. et al. N. Engl. J. Med. 351, 884-892 (2004)

9. Zhu, Y. et al. Pediatr. Res. 64, 154-158 (2008)

COMPANIES AND INSTITUTIONS MENTIONED

American Academy of Pediatrics, Elk Grove Village, III.

AstraZeneca plc (LSE:AZN; NYSE:AZN), London, U.K.

Baylor College of Medicine, Houston, Texas 


\section{ANALYSIS/PROSPECTS}

Discovery Laboratories Inc. (NASDAQ:DSCO), Warrington, Pa. GlaxoSmithKline plc (LSE:GSK; NYSE:GSK), London, U.K.

Medlmmune LLC, Gaithersburg, Md.

National Jewish Health, Denver, Colo.

Nationwide Children's Hospital, Columbus, Ohio

\section{TARGETS \& MECHANISMS}

Trellis Bioscience Inc., South San Francisco, Calif.

The Scripps Research Institute, La Jolla, Calif.

The University of Texas Southwestern Medical Center at Dallas,

Dallas, Texas

Winthrop-University Hospital, Mineola, N.Y. 